\title{
Where Does the Energy Loss Lose Strength?
}

\author{
G G Barnaföldi ${ }^{12}$, G Fai ${ }^{1}$, P Lévai ${ }^{2}$, G Papp ${ }^{3}$, B A Cole ${ }^{4}$ \\ ${ }^{1}$ CNR, Kent State University, Kent, OH 44242, USA \\ 2 RMKI KFKI, P.O. Box 49, Budapest, H-1525, Hungary \\ ${ }^{3}$ Eötvös University, Pázmány P. Sétány 1/A, Budapest, H-1117, Hungary \\ ${ }^{4}$ Nevis Laboratory, Columbia University, New York, NY, USA \\ E-mail: bgergely@rmki.kfki.hu
}

\begin{abstract}
Nuclear modification factors for pion production in $A u A u$ and $C u C u$ collisions are analyzed at very high transverse momenta. At $p_{T} \gtrsim 10 \mathrm{GeV} / \mathrm{c}$, the $R_{A A}\left(p_{T}\right)$ is determined mostly by the initial state nuclear modifications (e.g. EMC effect) and the non-Abelian jet-energy loss in the final state. At high momenta these effects together are strong enough to suppress $R_{A A}\left(p_{T}\right)$ to below 1 at RHIC energies. We display results using HKN shadowing in our pQCD improved parton model. Result of a similar calculation at LHC energies for $\mathrm{PbPb}$ collisions are also displayed. Based on $\mathrm{d} N / \mathrm{d} y$ estimates, a larger opacity value, $L / \lambda_{g} \approx 10 \pm 2$, is used for the produced partonic matter in central collisions at the LHC.
\end{abstract}

PACS numbers: 24.85.+p, 13.85.Ni, 13.85.Qk, 25.75.Dw

Submitted to: J. Phys. G: Nucl. Phys.

\section{Introduction}

Suppression of the inclusive pion spectra in central $A u A u$ collisions at RHIC relative to the peripheral (or $p p$ ) spectra can be understood in terms of non-Abelian energy loss [1, 2] in the quark-gluon plasma (QGP) created in central nuclear collisions [3, 4]. Early PHENIX data on the nuclear modification factor, $R_{A u A u}\left(p_{T}\right)$ explored the transverse momentum range $2 \mathrm{GeV} / \mathrm{c} \lesssim p_{T} \lesssim 10 \mathrm{GeV} / \mathrm{c}$, where the medium-induced non-Abelian relative energy loss was found to be almost constant as a function of $p_{T}$. A factor of $\sim 5$ suppression was measured [5, 6, 7], and a fractional energy loss, $S_{\text {loss }}$, was extracted [8].

The most recent (preliminary) high- $p_{T}$ data on $R_{A A}\left(p_{T}\right)$ [9] in central and midcentral $C u C u$ and $A u A u$ collisions cover the $p_{T}$ range up to $\approx 20 \mathrm{GeV} / \mathrm{c}$, and show a slight increase with $p_{T}$. Such a decrease of the strength of the energy loss is expected at very high transverse momenta, even though the EMC suppression is also present in this region[10].

In this paper we study the Gyulassy-Lévai-Vitev (GLV) 2] energy loss at high transverse momenta in central $A A$ collisions at RHIC and LHC energies. We concentrate 
on the $p_{T} \gtrsim 5 \mathrm{GeV} / \mathrm{c}$ region, where pQCD results can be trusted. To check the scaling properties, we present $R_{A u A u}$ as a function of both, $p_{T}$ and $x_{T}$. We expect the nuclear modifications to scale with $x_{T}$ and to lose strength at high opacity and large transverse momenta.

\section{Scaling of GLV Jet Quenching}

The slight increasing trend in the data on $R_{A A}\left(p_{T}\right)$ in Ref [9] beyond $p_{T} \gtrsim 10 \mathrm{GeV} / \mathrm{c}$ both in $A u A u$ and $C u C u$ collisions should be analyzed based on the high- $p_{T}$ behavior of nuclear shadowing and energy loss. The medium-induced non-Abelian energy loss, which is expected to be the stronger of the two effects, is described by

$$
\Delta E \approx \frac{C_{R} \alpha_{s}}{N(E)} \cdot \frac{L^{2} \mu^{2}}{\lambda_{g}} \cdot \log \left[\frac{E}{\mu}\right] \approx \frac{C_{R} \alpha_{s}}{N(E)} \cdot \frac{1}{A_{\perp}} \frac{\mathrm{d} N}{\mathrm{~d} y} \cdot\langle L\rangle \cdot \log \left[\frac{E}{\langle\mu\rangle}\right],
$$

where the meaning of the various symbols can be found in the GLV reference[2]. Most important for the present study is the fact that the implied relative energy loss, $\Delta E / E$, has a maximum at around $p_{T} \sim 5 \mathrm{GeV} / \mathrm{c}$ (which depends on the parameters weakly), and $\Delta E / E$ is almost constant in the $2 \mathrm{GeV} / \mathrm{c} \lesssim p_{T} \lesssim 10 \mathrm{GeV} / \mathrm{c}$ energy region for opacity values $L / \lambda_{g} \lesssim 2-4$ (see Ref. [4]).
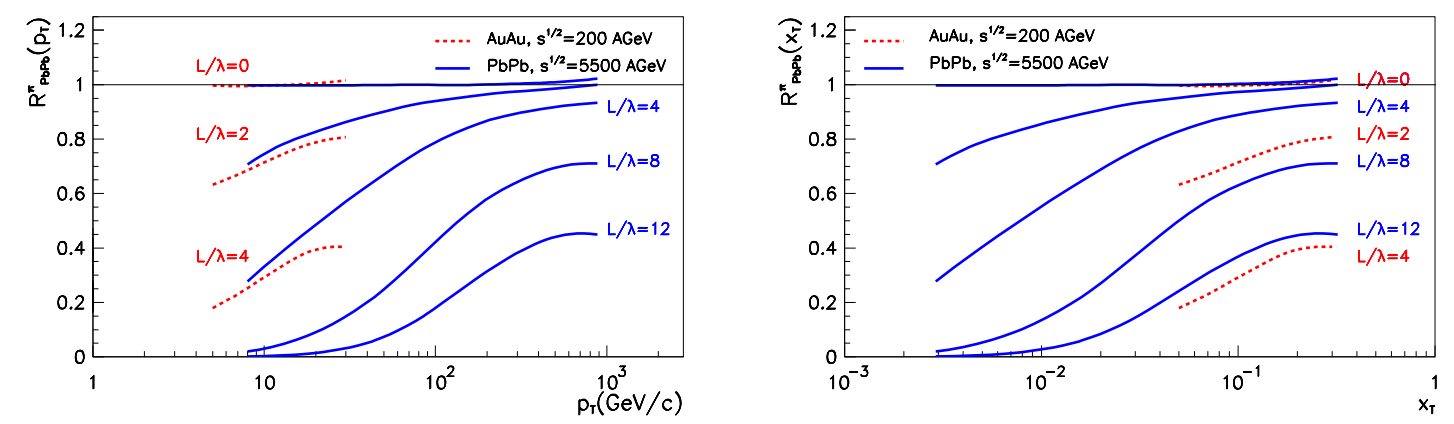

Figure 1. Scaling of energy loss effects in most central $A u A u$ and $P b P b$ collisions with $p_{T}$ (left panel) and with $x_{T}$ (right panel).

At higher opacities the maximum is relatively stable at around $E \sim 4-5 \mathrm{GeV}$, and it becomes more well-defined with increasing opacity. The effect of jet quenching thus becomes very strong around its maximum. At the same time, the energy loss is no longer a constant, but becomes an $E$-dependent quantity. Due to the $\sim \log (E / \mu)$ tail of the distribution, the energy loss of highly energetic jets is getting weaker, thus less suppression is expected at high $p_{T}$ values. Theoretical expectation for $\mathrm{d} N / \mathrm{d} y$ give $1200 \pm 300$ for the most central $A u A u$ collisions at RHIC [2, 4, 9], corresponding to $L / \lambda_{g} \approx 4$, while $\mathrm{d} N / \mathrm{d} y \sim(1500-4000)$ is a rough estimate for LHC energies, making $L / \lambda_{g}$ up to 12 relevant for calculations at LHC energies [11. 
To see the effect of the energy loss clearly, we present in Fig. 1 the generated nuclear modification with all initial and other final state nuclear effects switched off, using the above values. The left panel shows $R_{A A}^{\pi}\left(p_{T}\right)$, while in the right panel we display $R_{A A}^{\pi}\left(x_{T}\right)$.

With opacity $L / \lambda_{g}=0$, the nuclear modification is naturally $\sim 1$. The strongest suppression is associated with the highest density of the medium, reflected here in $L / \lambda_{g}=8-12$. It should be kept in mind that, due to the different energy densities at RHIC and LHC, a larger opacity value at LHC is expected to lead to a suppression similar to the one obtained at RHIC with a smaller $L / \lambda_{g}$. Comparing the left and right panels of Fig. 1, we conclude that $x_{T}$-scaling of $R_{A A}$ from RHIC (dashed lines) to LHC (solid lines) energies is more adequate than $p_{T}$ scaling.
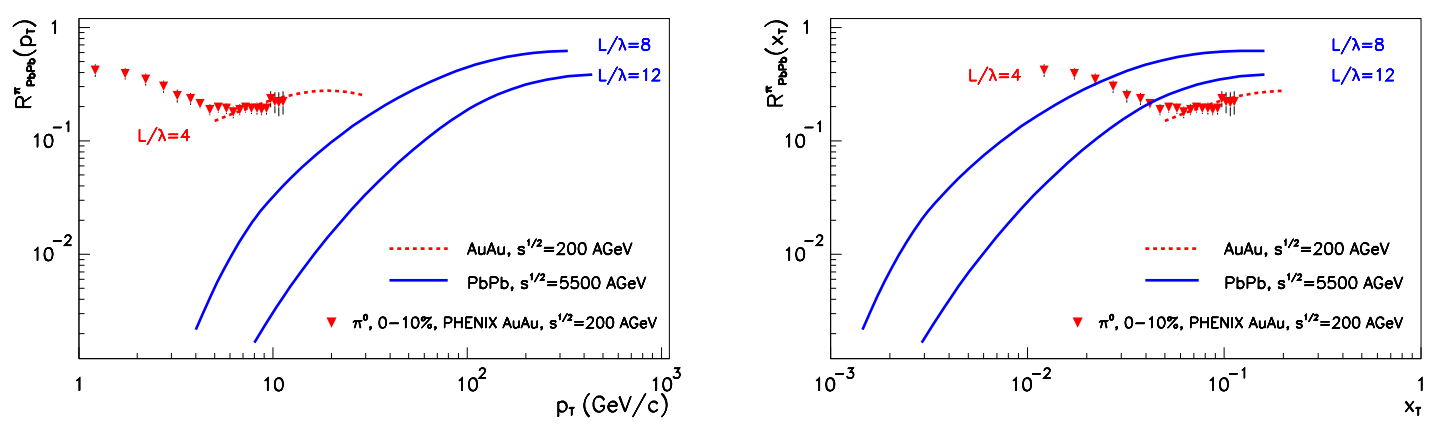

Figure 2. Calculations and data for nuclear modifications in most central $A u A u$ and $\mathrm{PbPb}$ collisions including final and initial state effects. (See text for details.)

Fig. 2 includes the initial state nuclear effects, using HKN [12] shadowing in the pQCD improved parton model [13. (The notation is similar to that of Fig. 1,) It can be seen from the left panel that after a minimum, the data on $R_{A A}$ increase toward high $p_{T}$, due to the logarithmic tail of the energy loss. At $p_{T} \gtrsim 10 \mathrm{GeV} / \mathrm{c}$ the calculated nuclear modification factor exhibits a maximum and then decreases again, reaching the EMC region. As seen on the right panel, the convolution with the shadowing function preserves the $x_{T}$ scaling of the nuclear modification [14]. Based on the scaling, we attribute an approximate opacity parameter $\sim 10 \pm 2$ to central $\mathrm{PbPb}$ collisions at $\sqrt{s}=5.5$ ATeV LHC energy. Note, however, that in minimum bias measurements an averaged opacity should be relevant, much smaller than the central maximum value. It should also be remembered that the suppression has an angular dependence, which needs to be tested by further geometrical studies of jets.

\section{Concluding Remarks}

We studied the non-Abelian jet energy loss in the first-order GLV framework. Concentrating on the perturbative, high- $p_{T}$ region, we pointed out that the maximum 
of the energy loss becomes sharper, and that the phenomenon loses strength due to the logarithmic tail at high $p_{T}$ and opacities greater than 4. At larger opacities the standard picture of a constant relative energy loss needs to be modified. Thus, energy-loss effects are more complicated than a simple shift of the high- $p_{T}$ spectra. This may explain why $S_{\text {loss }}$ does not show perfect scaling for different system sizes or centralities [9].

We found that the energy loss scales with $x_{T}$ (using appropriately different opacity values), and the introduction of initial-state nuclear effects preserves this scaling. Based on Fig. 1 we extract the opacity parameter $\sim 10 \pm 2$ for most central $\mathrm{PbPb}$ collisions at $\sqrt{s}=5.5$ ATeV LHC energy.

The extracted $L / \lambda_{g}$ values are high, but should only appear in the most dense region of the collision. The present description of the jet is incomplete, since the geometrical

properties of the jet were not taken into account. A detailed analysis should investigate the angular size variation of the jet and the angular distribution of the energy loss inside the jet.

\section{Acknowledgments}

One of the authors (GGB) would like to thank to the organizers for local support. Our work was supported in part by Hungarian OTKA PD73596, T047050, NK62044, and IN71374, by the U.S. Department of Energy under grant U.S. DE-FG02-86ER40251, and jointly by the U.S. and Hungary under MTA-NSF-OTKA OISE-0435701.

\section{References}

[1] Bayer R et al. 1997 Nucl. Phys. B 483 291, 484 265, 531403

[2] Gyulassy M, Lévai P, and Vitev I 2000 Phys. Rev. Lett. 85 5535, Nucl. Phys. B 571197

[3] David G et al. (PHENIX) 2002 Nucl. Phys. A 698227

[4] Lévai P et al. 2002 Nucl. Phys. A 698631

[5] Adcox K et al. (PHENIX) 2003 Phys. Lett. B 561 82, 2002 Phys. Rev. Lett. 88022301

[6] Adler S S et al. (PHENIX) 2003 Phys. Rev. Lett. 91072301

[7] Barnaföldi G G et al. Proc. of 32 ${ }^{\text {nd }}$ Int. Symposium on Multiparticle Dynamics (ISMD 2002) 145, Alushta 2002

[8] Adler S S et al. (PHENIX) 2007 Phys. Rev. C 76034904

[9] Adare A et al. (PHENIX) 2008 arXiv:0801.1665, 0801.4555, 0801.4020

[10] Cole B A, Barnaföldi G G, Levai P, Papp G and Fai G 2007 arXiv:hep-ph/0702101.

[11] Arnesto N (ed.) 2008 J. Phys. G 35054001

[12] Hirai M, Kumano S, Nagai T-H and Sudoh K 2007 Phys. Rev. D 75094009

[13] Zhang Y, Fai G, Papp G, Barnaföldi G G and Lévai P 2002 Phys. Rev. C 65034903

[14] Barnaföldi G G et al. 2008 Cold Nuclear Modifications at RHIC and LHC-poster at Quark Matter 2008 to be published in Indian Journal of Physics 